\title{
Depressão entre estudantes de dois cursos de enfermagem: autoavaliação da saúde e fatores associados*
}

\author{
Depression among students from two nursing undergraduate programs: self-assessment on health and associated factors \\ Depresión entre estudiantes de dos cursos de pregrado en enfermería: autoevaluación de salud y factores asociados
}

\author{
Antonia Regina Ferreira Furegato', Jair Lício Ferreira Santos", Edilaine Cristina da Silval \\ 'Universidade de São Paulo. Escola de Enfermagem de Ribeirão Preto. \\ Departamento de Enfermagem PsiQuiátrica e Ciencias Humanas. Ribeirão Preto, SP \\ "Universidade de São Paulo. Faculdade de Medicina de Ribeirão Preto. Departamento de Medicina Social. Ribeirão Preto, SP
}

Submissão: $12 / 12 / 2008$

\section{RESUMO}

Objetivou-se verificar a prevalência de depressão, auto-avaliação da saúde e fatores associados entre estudantes de enfermagem. PesQuisa realizada em Ribeirão Preto com estudantes do $2^{\circ}$ ano de 2 cursos de graduação. Instrumentos: Critérios de Classificação Econômica Brasil; Inventário de Depressão de Beck; Avaliação da Qualidade de Vida. Respeitou-se os procedimentos éticos. Dados analisados bi e multivariada. Nos 114 sujeitos predominam mulheres e solteiros. Entre os 65 do Bacharelado predominam níveis econômicos A2, B1 e B2, 69,6\% não trabalham, 32\% abaixo dos 20 anos de idade. Nos 49 da Licenciatura predominam níveis B2 e C, 75,8\% têm trabalho remunerado, 32,6\% estão acima dos 25 anos. Depressão presente em 15,4\% no Bacharelado e 28,6\% na Licenciatura. Decresce a depressão conforme cresce o nível econômico na Licenciatura. Auto-avaliação mental ao estudante e curso em Que está matriculado tiveram as razões de prevalência significativas para depressão. Resultados compatíveis com estudos de depressão entre jovens.

Descritores: Depressão; Enfermagem; Estudantes de enfermagem; Qualidade de vida.

\section{ABSTRACT}

This study aimed to verify the prevalence of depression and associated factors among undergraduate nursing students. Descriptive research carried out in Ribeirão Preto with students from two undergraduate programs. Instruments: Brazilian Economic Classification Criteria; Beck Depression Inventory; Quality of Life Assessment. It was performed bivariate and multivariate analysis of data. Ethical procedures were respected. Among the 114 subjects, most are women and single. Bachelor Program: $32 \%$ are aged less than 20 years old, from economic levels A2, BI and B2 and 69,6\% do not work. Teaching Diploma Program: 32,6\% older than 25 years old, levels B2 and $\mathrm{C}$ and $75,8 \%$ work. Depression detected in 15,4\% of the students at Bachelor's Program and 28,6\% from the Teaching Diploma Program. The percentage of depression decreases as Teaching Diploma Program students' economical level increases. Mental self assessment carried out by student and program enrolled had significant prevalence rates.

Key words: Depression; Nursing, students; Quality of life.

\section{RESUMEN}

Este estudio tuvo como objetivo verificar la prevalencia de depresión y factores asociados entre estudiantes de enfermería. Investigación descriptiva, realizada en Ribeirão Preto con estudiantes de 2 carreras de pregrado. Instrumentos: Criterios de Clasificación Económica Brasileño; Inventario de Depresión de Beck; Evaluación de la Calidad de Vida. Los procedimientos éticos fueron respectados. Se hizo análisis bivariada y multivariada de los datos. Entre los 114 sujetos predominan mujeres y solteros. Licenciatura: $32 \%$ tienen menos de 20 años, niveles económicos A2, B I y B2 y 69,6\% no trabajan. Bachillerato: 32,6\% tienen más de 25 años, niveles B2 y C y 75,8\% trabajan. Depresión fue detectada en $15,4 \%$ de los estudiantes de Licenciatura y 28,6\% de Bachillerato. La depresión decrece conforme crece el nivel económico de los alumnos de Bachillerato. Autoevaluación mental hecha por estudiantes y curso en Que esta registrado tuvieron tasas de prevalencia significativas para la depresión.

Descriptores: Depresión; Enfermería, estudiantes; Calidad de vida.

*Projeto universal financiado pelo CNPQ no 47207/2006-I

AUTOR CORRESPONDENTE Antonia Regina Ferreira Furegato. Av. Bandeirantes, 3900. Escola de Enfermagem de Ribeirão Preto. CEP 14040-920. Ribeirão Preto, SP. E-mail: furegato@eerp.usp.br 


\section{INTRODUÇÃO}

Os transtornos depressivos produzem um pesado ônus para a pessoa, para sua família e para a sociedade. Estudos epidemiológicos revelam a relação de perda de produtividade e faltas à escola, ao trabalho e outros compromissos sociais. Projeções indicam Que, em 2020, o custo global medido em termos de anos de vida, ajustados por incapacitação, ficarão atrás somente das doenças cardíacas e isquêmicas ${ }^{(1-5)}$. Entre as diversas afecções crônicas de alto custo pessoal e social a depressão é uma doença tratável e curável, devido aos avanços e novas opções terapêuticas. Apesar disto, nem todas as pessoas afetadas recebem o devido tratamento e orientação ${ }^{(2)}$. Estudos documentam redução dos gastos com saúde Quando a depressão foi diagnosticada e adequadamente tratada ${ }^{(6)}$.

Por uma série de fatores como a desinstitucionalização, a reordenação dos serviços de saúde, o deslocamento político do foco decisório, a introdução de novos antidepressivos, a participação mais ativa da comunidade, a preocupação com os custos pessoais e sociais da doença, bem como os custos com os tratamentos cresce a necessidade de evidenciar o alcance desse conjunto de fatores e suas conseQüências para a população atingida pela doença mental.

Pesquisas mostram Que há relação entre a presença, a intensidade e o comprometimento da depressão com o funcionamento físico, psicológico, social e ambiental ${ }^{(7-8)}$.

A depressão tem também relação com a Qualidade de vida da pessoa afetada. Numa revisão de Quase todos os fatores acima mencionados, chama-se a atenção para a ligação da depressão com a Qualidade vida das pessoas afetadas ${ }^{(4)}$.

A integração entre tratamento psiQuiátrico e reabilitação psicossocial onde se vive um processo de respostas positivas dos profissionais e da sociedade promovendo ações de reconhecimento do doente mental como cidadão ajuda os mesmos a atingirem seus melhores níveis de funcionamento e de Qualidade de vida ${ }^{(3,9-13)}$.

A depressão ocorre em todas as idades; está se tornando mais frequente entre os jovens? As intensas reações emocionais ocasionais e próprias desta fase são claramente diferenciadas da presença de transtorno depressivo?

Os critérios de classificação dos transtornos depressivos na juventude são os mesmos dos adultos. As principais diferenças são Que o humor do jovem pode ser "irritável" ou "deprimido" e mais reativo. Por outro lado, as terapias cognitivo-comportamentais são mais eficazes para jovens ${ }^{(14-15)}$.

É importante investigar o impacto da presença de sintomatologia depressiva sobre o funcionamento cotidiano do jovem, e, verificar planos de suicídio, perda de peso acentuada e falta de energia, considerando-se a duração e a evolução destes estados no diagnóstico clínico ${ }^{(3,5,12,15)}$.

Tendo em vista o grande custo da depressão e suas conseQüências é importante detectar precocemente a presença de depressão entre os estudantes de enfermagem e entre os enfermeiros visando seu desempenho pessoal e profissional ${ }^{(4)}$.

Estudos realizados entre enfermeiros e estudantes de enfermagem registraram manifestações físicas e emocionais de desgaste, Queixas sobre as condições de trabalho, dificuldades em suas relações interpessoais, agravadas pela convivência diuturna com o sofrimento e a dor ${ }^{(16-19)}$.

Na função de professores de enfermagem psiQuiátrica, a saúde mental dos jovens alunos preocupa tanto Quanto a Qualidade do ensino que lhes é ministrado. Portanto, partimos do pressuposto de que o enfermeiro, além de bem preparado cientifica e tecnicamente, deve gozar de boa saúde mental para oferecer os melhores cuidados aos portadores de depressão.

\section{OBJETIVO}

Verificar a prevalência de depressão entre estudantes de dois cursos de Graduação em Enfermagem (Bacharelado e Licenciatura) e fatores sócio demográficos associados.

\section{METODOLOGIA}

Este estudo faz parte do NUPRI - Núcleo de Pesquisas e Ensino das Relações Interpessoais em Enfermagem (CNPQ no 305698/ 2006-0). Um dos subprojetos deste núcleo de pesquisas, aprovado no edital Universal $n^{\circ} 472070 / 2006-1$, focaliza estudos de depressão identificando conhecimento, opinião sobre o tema e investiga outras variáveis como Qualidade de vida e auto-estima Que possam influenciar na presença da depressão entre grupos como estudantes de enfermagem, enfermeiros e mulheres em diferentes contextos de sua vivência. Estes estudos pretendem contribuir para o melhor cuidado de enfermagem às pessoas Que sofrem pela convivência com a depressão.

A realização desta pes@uisa foi precedida pela avaliação do projeto completo pelo Comitê de Ética em Pesquisa da Escola de Enfermagem de Ribeirão Preto/USP, com aprovação no 0 1 4/2002.

Os estudos de depressão, desenvolvidos pelos pesquisadores do NUPRI, consideram e utilizam toda diversidade de métodos e técnicas de investigação necessários à execução das suas pesQuisas. Dessa forma, recorre-se tanto a técnicas Qualitativas como Quantitativas bem como a interface entre as mesmas. O contexto histórico, vivencial, relacional e também os estudos científicos divulgados na literatura sobre este tema permitem contextualizar achados e sustentar a discussão teórica de nossas pesquisas.

A medida escalar, utilizada nestes estudos, constitui-se uma das várias formas de medida Que a psicometria pode assumir, incluindo os testes psicológicos, os inventários, os Questionários e as escalas Que apresentam os parâmetros necessários para Que sejam instrumentos legítimos e válidos ${ }^{(20)}$.

A pesquisa foi realizada na Escola de Enfermagem de Ribeirão Preto, em 2007, junto aos alunos do Curso de Graduação em Enfermagem, do $2^{\circ}$ ano letivo, tanto do Bacharelado (B) como da Licenciatura (L). Os cursos de Graduação em enfermagem têm previsão curricular para serem completados entre 4 e 5 anos letivos.

No curso de enfermagem diurno (Bacharelado) ingressam anualmente 80 alunos sendo esta a $53^{\text {a }}$ turma.

O curso de Licenciatura (vespertino/noturno) iniciou-se em 2006, com ingresso de 50 alunos, anualmente, sendo esta a primeira turma, cursando, portanto, o $2^{\circ}$ ano letivo.

A amostra limitou-se aos alunos do $2^{\circ}$ ano dos dois Cursos de graduação porQue não havia turmas mais adiantadas na Licenciatura. Com este critério, torna-se viável a comparação dos dois grupos já Que um curso é diurno (Bacharelado) e outro vespertino/noturno (Licenciatura).

Havia 85 alunos do Bacharelado matriculados na disciplina 
escolhida para a coleta dos dados. Como era disciplina teórica esperava-se obter o máximo de participação dos sujeitos. Entretanto, participaram 65 sujeitos $(76,5 \%)$ pois três alunos se negaram a participar e os demais 17 não compareceram à sala de aula no dia da aplicação dos testes.

Obteve-se a totalidade dos sujeitos do curso de Licenciatura, ou seja, dos 49 matriculados todos responderam aos testes, antes da aula de ações clínicas em saúde mental.

Utilizou-se, neste estudo, um conjunto de instrumentos conhecidos, validados e largamente utilizados no meio científico: CEB, IDB e WHOQOL.

CEB - Critérios de Classificação Econômica Brasil: Construído para definir grandes classes Que atendam as necessidades de segmentação (por poder aQuisitivo), utiliza-se de técnicas estatísticas baseadas no coletivo. Para o grupo pesquisado é baixa a probabilidade de erro de classificação ${ }^{(21)}$. O aluno anotou a Quantidade dos itens existentes na casa da sua família de origem e o grau de instrução do chefe da família.

- IDB - Inventário de Depressão de Beck: Escala com 21 itens cuja intensidade varia de 0 a 3 , representando sintomas e atitudes tais como tristeza, pessimismo, sensação de fracasso, falta de satisfação, sensação de culpa e de punição, auto depreciação, retração social, indecisão, distorção da imagem corporal, inibição para o trabalho, distúrbios do sono, fadiga, perda de apetite e de peso, preocupação somática e diminuição da libido ${ }^{(22)}$.

As propriedades psicométricas da versão em português foram testadas avaliando-se diferenças fatoriais nas dimensões: cognição - afeto, auto-depreciação e somática e a discriminação da depressão em jovens ${ }^{(23)}$.

Identifica de modo confiável os casos de depressão sendo adequada para estudos epidemiológicos e clínicos, de uso fácil e baixo custo, permitindo comparações e avaliações ${ }^{(8)}$. É um teste auto-aplicado, especialmente, entre sujeitos como os estudantes universitários.

Os índices de classificação do IDB são diferentes da clínica caso seja aplicado como rastreamento numa população não suspeita (como é o caso dos alunos de enfermagem) buscando sinais indicativos de depressão. No rastreamento considera-se: menos de 15 = sem depressão; 15 a $19=$ disforia (depressão leve); de 20 a 29 = depressão moderada; acima de 30 - depressão grave.

- Avaliação de Qualidade de vida - WHOQOL: Instrumento genérico, de caráter subjetivo e multidimensional, destinado a avaliar a percepção das pessoas em relação a sua própria vida, saúde, sexualidade e ambiente informando desde seu bem estar físico até a realização espiritual. A escala completa foi desenvolvida num projeto multicêntrico, coordenado pela OMS, sendo então adaptada ao português ${ }^{(24-25)}$.

A WHOQOL (versão reduzida) consta de 26 Questões Que representam facetas dos domínios contidos no instrumento original (físico, psicológico, relações sociais e meio ambiente). Duas Questões referem-se a aspectos gerais da Qualidade de vida e saúde. São atribuídos 1 e 2 pontos às categorias de respostas desfavoráveis, 3 às intermediárias e 4 e 5 pontos às favoráveis.

Tanto no Inventário de Depressão de Beck (IDB) como no Escala de avaliação de Qualidade de vida (WHOQOL reduzido) há cabeçalhos onde o sujeito informa alguns dados pessoais e sociodemográficos.
Após terem sido devidamente esclarecidos Quanto aos aspectos éticos, procedimento para respostas e importância da sua participação, garantindo sua liberdade de opção, os estudantes dos cursos de graduação em Enfermagem (diurno e vespertino) responderam ao conjunto de testes (escalas e Questionários). Todos assinaram o Termo de Consentimento Livre e Esclarecido.

Os alunos responderam, seguindo as instruções dos testes, com o respaldo de dois pesquisadores e um aluno de Iniciação Cientifica para esclarecimento das dúvidas individualmente.

Garantiu-se também a rápida devolução dos resultados para os sujeitos, especialmente no Que se referia à identificação da depressão. Os alunos identificados com sinais de depressão moderada e grave foram chamados para atendimento, tendo sido orientados individualmente.

Nas análises de possíveis associações entre as variáveis foi utilizado o Teste Exato de Fisher. Para comparação dos escores de Qualidade de vida entre vários grupos utilizou-se o teste de KruskalWallis e para a comparação de escores entre dois grupos o teste de Mann Whitney ${ }^{(26)}$

Com o intuito de averiguar as possíveis relações das variáveis em seu conjunto, com a presença ou não de depressão, procedeuse a uma análise multivariada utilizando-se a regressão de Poisson com estimativas robustas da variância, com a variável desfecho (Depressão) formada pelas categorias zero (ausência de Depressão) e um (Presença de Depressão). Para facilidade de interpretação, as variáveis independentes foram igualmente transformadas em dicotômicas e a estatística utilizada para comparação foi a "razão de prevalência”. O ajuste foi realizado através do STATA 9.2 e anotados os seus principais parâmetros ${ }^{(27-28)}$.

Definição das variáveis para o ajuste de regressão logística: as variáveis independentes foram selecionadas entre as disponíveis, Quanto às características sociodemográficas, auto avaliação da saúde e curso frequentado. Todas essas variáveis foram avaliadas binomialmente, de forma a facilitar a análise dos resultados. As definições, categorias de referência e de contraste encontram-se agrupadas no Quadro I.

\section{RESULTADOS}

Dos 120 alunos matriculados no $2^{\circ}$ ano do Curso de Graduação em Enfermagem, participaram 114 sendo todos os alunos matriculados no curso noturno - Licenciatura (49) e 76,5\% dos matriculados no curso diurno - Bacharelado (65 alunos).

Como se esperava, a grande maioria dos estudantes era do sexo feminino, o Que representa $82,5 \%$. Entretanto, a presença masculina com $17,5 \%$ excede informações registradas na literatura ${ }^{(18,29)}$. A maioria dos alunos (104), nos dois cursos de enfermagem, são solteiros; havia oito casados e dois casos de mulheres separadas.

Vale ressaltar Que $88,3 \%$ das mulheres estão abaixo dos 24 anos e $85 \%$ dos homens acima dos 20 anos. Dezoito alunos têm mais de 25 anos de idade, dentre eles sete homens. No conjunto, $62,3 \%$ dos alunos estão na faixa entre 20 e 24 anos de idade, destacando-se que 32,6\% da Licenciatura estão acima dos 25 anos e 32\% do Bacharelado, abaixo dos 20 anos.

Observou-se relação inversa referente à ocupação remunerada nos dois cursos: no Bacharelado $69,6 \%$ não trabalham e, na Licenciatura $75,8 \%$ trabalham possivelmente porque o curso de 


\begin{tabular}{|c|c|c|c|}
\hline \multicolumn{4}{|c|}{ VARIÁVEIS INDEPENDENTES } \\
\hline NOME & DEFINIÇÃO & REFERÊNCIA & CONTRASTE \\
\hline Idade & Idade em anos & Até 20 anos & Acima de 20 anos \\
\hline Saude Física & Auto avaliação da saúde física & Muito boa/ Boa & Média /Fraca/Ruim \\
\hline Saúde Mental & Auto avaliação da saúde mental & Muito boa/ Boa & Média /Fraca/Ruim \\
\hline Sexo & Sexo & Feminino & Masculino \\
\hline Trabalho & Exerce ocupação remunerada & Sim & Não \\
\hline Curso & Curso fręuentado & Bacharelado & Licenciatura \\
\hline Classe Social & $\begin{array}{c}\text { Classe definida pelo Critério Sócio } \\
\text { econômico Brasil }\end{array}$ & $\mathrm{A} 2$ & $\mathrm{~B} 2 / \mathrm{bl} / \mathrm{C} / \mathrm{D}$ \\
\hline \multicolumn{4}{|c|}{ VARIÁVEL RESPOSTA } \\
\hline Depressão & $\begin{array}{l}\text { Presença de depressão (leve, } \\
\text { moderada ou grave) }\end{array}$ & Não & Sim \\
\hline
\end{tabular}

Quadro 1. Definições, referências e contrastes das variáveis independentes utilizadas na análise.

Licenciatura oferece mais oportunidades de horários para Que os estudantes possam trabalhar.

Depressão, Percepção de saúde e variáveis sócio-demográficas

Com base nas respostas dos 114 alunos Que freeüentam o $2^{\circ}$ ano dos cursos de enfermagem da EERP/USP ao Inventário de Depressão de Beck, os resultados mostram Que há 15,4\% do Bacharelado (curso diurno) e $28,6 \%$ da Licenciatura (curso noturno) com sinais indicativos de depressão (leve, moderada e grave) dos Quais $14 \%$ podem ser classificados como depressão moderada (6) e grave (3).

O resultado do teste de Classificação Econômica Brasil (CEB) não registrou nenhum sujeito na classe $\mathrm{A} I$ nem na classe $\mathrm{E}$. Portanto, estes alunos não são muito pobres, nem muito ricos. No curso diurno, a concentração registrou-se nos níveis A2, BI e B2 (86\%). $\mathrm{O}$ teste exato de Fisher não evidenciou correlação significativa entre classe social e depressão, porém vale ressaltar algumas semelhanças e diferenças entre os dois grupos.

Nestes três níveis estão alocados todos os 10 casos de alunos com sinais indicativos de depressão do Bacharelado. Também, não foi identificado nenhum caso de depressão entre os alunos das classes C e D, apesar de haver nove deles (14\%) nestas classes.

Entre os alunos da Licenciatura, entretanto, observou-se uma tendência para os níveis mais baixos, diferente do Bacharelado Que tende para os níveis mais altos. Observe-se na Figura I Que nos níveis B2 e C encontram-se $67 \%$ dos alunos do curso noturno, concentrando-se também (nestes 2 níveis) a presença de sinais indicativos de depressão (10 dos 114 ). Observou-se que decresce a presença de depressão, conforme cresce a classe social entre os alunos da Licenciatura.

No cômputo geral, 7 I\% dos chefes das famílias dos alunos têm bom nível de instrução, ou seja, nível superior incompleto (36\%) ou superior completo (35\%).

Apenas um dos oito alunos do Bacharelado que têm trabalho remunerado apresenta sinais de depressão leve. Na licenciatura também não se encontrou depressão grave entre os alunos Que trabalham. Entretanto, dos 25 Que trabalham, o Que representa $51 \%$ do total, seis (12\%) têm sinais indicativos de depressão leve ou moderada. Interessante observar Que naQueles alunos Que não têm sinais de depressão na Licenciatura (71\%), 19 exercem ocupação remunerada e 16 não, o Que reforça a falta de correlação entre as variáveis depressão $x$ trabalho + estudo.

No cabeçalho da WOQOL, além dos dados sociodemográficos solicita-se ao sujeito Que informe como está sua saúde física e mental. Os alunos do curso diurno (sendo apenas um deles com depressão) acham sua saúde física ruim e nenhum dos 69 considera sua saúde mental ruim. Por outro lado, nenhum deles acha sua saúde física ou mental muito boa entre os 10 Que têm depressão.

A grande maioria dos alunos do curso diurno classifica sua saúde física como média ou boa. Entretanto, a auto-avaliação da saúde mental apresentou correlação significativa $(0,001)$ visto Que está associada à presença de depressão (6 dos 10 casos avaliaram sua saúde mental fraca ou média).

Entre os alunos do curso noturno (Licenciatura), as correlações foram significantes para o teste de Fisher nas 2 situações $(0,000$ e 0,010 ). Dos que não tinham sinais de depressão, $65 \%$ consideram sua saúde física boa ou muito boa e $55 \%$ consideram sua saúde mental boa ou muito boa. Entretanto, entre os alunos com sinais de depressão, metade deles percebe que tanto a saúde física como a saúde mental não é boa. Observe-se Que neste grupo também ninguém avalia sua saúde física ou mental como sendo ruim.

$\mathrm{Na}$ avaliação da Qualidade de vida dos 2 grupos de alunos observou-se Que há associação com a presença de depressão. Os piores níveis de Qualidade de vida nos domínios físico, psicológico e social entre os alunos do Bacharelado foram registrados pelos sujeitos Que têm depressão, conforme indicou o teste de Kruskal Wallis. Entre os alunos da Licenciatura, todos os resultados foram significantes no Kruskal Wallis, inclusive no domínio ambiental (Tabela 1).

O teste de Kruskal Wallis, para classe social e Qualidade de vida, mostrou Que os resultados não foram significativos. Portanto, o Que marca a diferença encontrada na comparação da depressão e Qualidade de vida não é a classe social.

Através do teste Mann Whitney, com nível de significância de 5\% comparou-se as medianas dos escores de Qualidade de vida entre os alunos dos dois cursos de enfermagem, nos 4 domínios. 


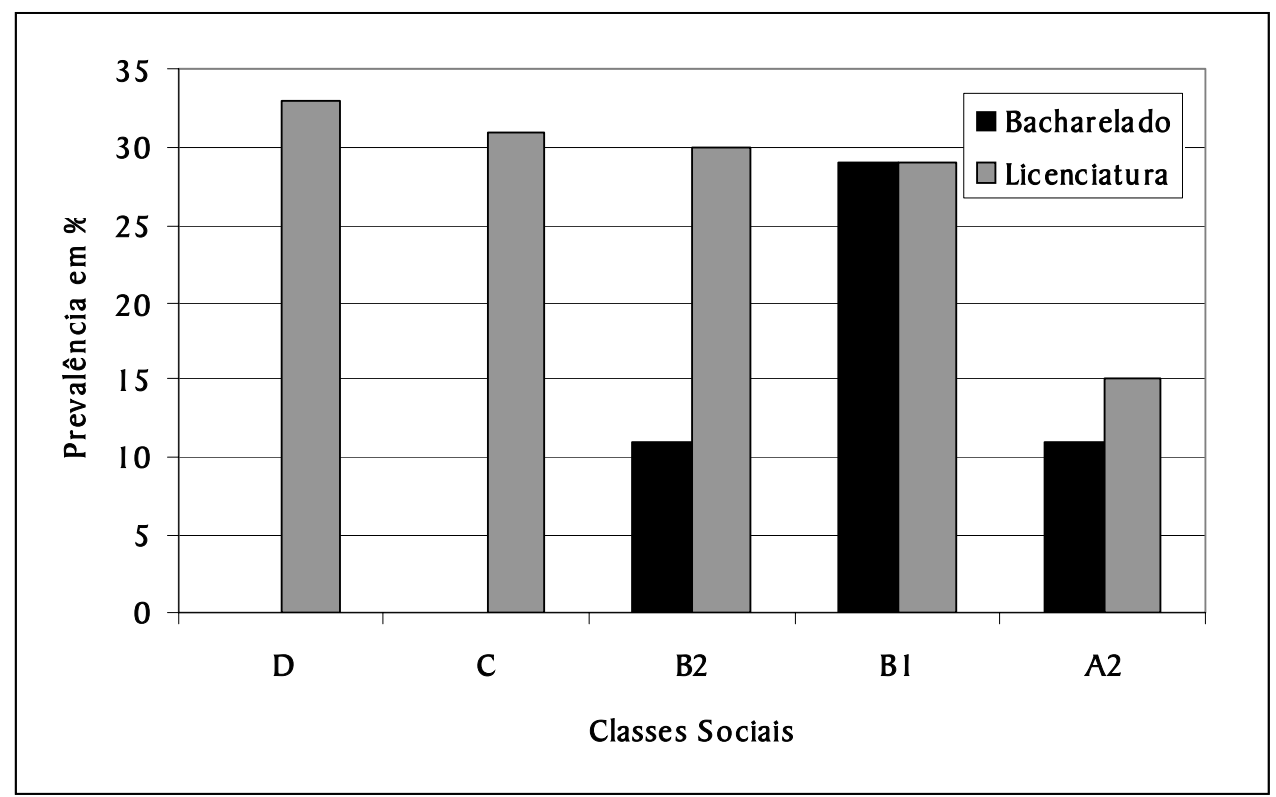

Figura 1. Prevalência de depressão por curso e classe social. Ribeirão Preto, 2008.

Tabela 1. Medianas dos escores de níveis de depressão e eualidade de vida em cada domínio para o Curso de Enfermagem Bacharelado e Licenciatura Ribeirão Preto, 2008.

\begin{tabular}{|c|c|c|c|c|}
\hline \multirow{2}{*}{$\begin{array}{l}\text { Classes de Depressão } \\
\text { Bacharelado }\end{array}$} & \multicolumn{4}{|c|}{ Medianas de Qualidade vida (domínios) } \\
\hline & Físico & Psicológico & Social & Ambiental \\
\hline Sem depressão & 64.3 & 62.5 & 75.0 & 62.5 \\
\hline Disforia & 50.0 & 47.9 & 58.3 & 54.7 \\
\hline Moderada & 39.3 & 37.5 & 25.0 & 59.4 \\
\hline Grave & 42.9 & 41.7 & 66.7 & 50.0 \\
\hline Total & 60.7 & 58.3 & 75.0 & 59.4 \\
\hline Teste de Kruskal Wallis & 12,8 & 18,6 & 12,5 & 7,7 \\
\hline $\begin{array}{l}\text { Qui Quadrado OBS } \\
\text { P (3GL) }\end{array}$ & 0,005 & 0,000 & 0,006 & 0,053 \\
\hline \multicolumn{5}{|l|}{ Licenciatura } \\
\hline Disforia & 50.0 & 54.2 & 66.7 & 53.1 \\
\hline Moderada & 46.4 & 33.3 & 33.3 & 40.6 \\
\hline Grave & 17.9 & 12.5 & 8.3 & 37.5 \\
\hline Total & 64.3 & 62.5 & 66.7 & 59.4 \\
\hline Teste de Kruskal Wallis & 23,6 & 24,0 & 18,2 & 14,7 \\
\hline $\begin{array}{l}\text { Qui Quadrado OBS p } \\
\text { (3GL) }\end{array}$ & 0,000 & 0,000 & 0,000 & 0,002 \\
\hline
\end{tabular}

Os resultados evidenciaram diferenças significantes apenas no domínio social para Qualidade de vida onde os sujeitos do Bacharelado têm Qualidade de vida superior (mediana $=75$ ) em relação aos da Licenciatura (mediana $=66,7$ ).

O teste de Mann Whitney para comparar a Qualidade de vida com ocupação remunerada, também não evidenciou diferenças significantes ao nível de 5\%.

Em função desses resultados, optou-se por uma análise multivariada afim de considerar o efeito de cada variável na presença das demais e em caso positivo, indicar algum índice preditivo. Os resultados do ajuste do modelo de regressão de Poisson é apresentado na Tabela 2 Que segue, com os respectivos parâmetros de ajuste e valores das estatísticas relevantes.

O teste de ajuste resultou em Qui Quadrado, com 104 graus de liberdade igual a 49,6 e P igual a 1.000 . Isto é, as predições do modelo não diferem dos valores observados. Apenas duas variáveis tiveram Razões de Prevalência (RP) significantes: a auto avaliação da saúde mental e o curso Que o sujeito frequenta.

A variável Saúde mental é a Que apresenta maior razão de prevalência $(5,82)$, isto é, os indivíduos que auto classificaram a 
Tabela 2. Resultados do ajuste da regressão de Poisson: razões de prevalência (RP) para cada variável, (prevalência do contraste em relação à referência); erros-padrão; valores da estatística $\mathrm{z}$ para o teste de significância, probabilidades de ocorrência P(z); e intervalos de confiança de 95\% para RP. Ribeirão Preto, 2008.

\begin{tabular}{lcccccc}
\hline \multicolumn{1}{c}{ Variáveis } & $\mathbf{R P}$ & $\begin{array}{c}\text { Estimativa } \\
\text { robusta do } \\
\text { erro padrão }\end{array}$ & $\mathbf{Z}$ & $\mathbf{P}>|\mathbf{z}|$ & \multicolumn{2}{c}{ Intervalos de Confiância(95\%) } \\
\cline { 5 - 7 } & 1,76 & 0,60 & 1,66 & 0,097 & Lim inf & Lim sup \\
\hline Saúde física & 5,82 & 2,79 & 3,68 & $0,000(*)$ & 2,28 & 3,4 \\
Saúde mental & 0,65 & 0,21 & $-1,33$ & 0,184 & 0,33 & 1,23 \\
Idade & 0,72 & 0,43 & $-0,55$ & 0,581 & 0,22 & 2,32 \\
Sexo & 0,79 & 0,29 & $-0,65$ & 0,515 & 0,38 & 1,6 \\
Trabalho & 2,11 & 0,66 & 2,39 & $0,017(*)$ & 1,14 & 3,89 \\
Curso & 0,80 & 0,64 & $-0,28$ & 0,783 & 0,17 & 3,85 \\
Classe social & & & & &
\end{tabular}

Parâmetros de ajuste:

$N=I / 2$ Wald chi2 $(7)=45.16(P<0,000)$ Log pseudoverossimilhança $=-47.8 I$

Pseudo R2 $=0.20$ (*) Resultados significantes a $5 \%$.

sua saúde mental como "média, fraca ou ruim" têm prevalência de depressão Quase seis vezes maior do Que Quem a classificou como "boa ou muito boa".

Outra variável Que resultou significante com o ajuste foi o Curso freqüentado: o aluno da Licenciatura (curso noturno) tem mais de duas vezes a prevalência de depressão do Que o aluno do Bacharelado.

\section{DISCUSSÃO}

Esta pesquisa partiu dos seguintes hipóteses: Que os alunos do curso noturno (Licenciatura) seriam de classe social mais baixa em relação ao Bacharelado (verificou-se Que é verdadeiro); Que a faixa etária da Licenciatura estaria acima dos 30 anos e que muitos tinham trabalho remunerado durante o dia (verdadeiros); Que haveria mais casos de depressão na Licenciatura (verdadeiro); Que haveria diferenças significativas entre Qualidade de vida dos 2 grupos de sujeitos (parcialmente verdadeira).

Estudos sobre a Qualidade de vida dos acadêmicos, residentes e profissionais de enfermagem os define como seres humanos Que optaram por ajudar outros seres humanos a nascerem, vivenciarem, superarem problemas e limitações e morrerem dignamente. Para realizar as ações Que integram seu trabalho enfrentam situações de sofrimento e estresse. Assim, ao mesmo tempo em Que influem no processo pessoal de resolução de situações cotidianas, estas vivências determinam condições e estilo de vida ${ }^{(16,18-19)}$.

Muitos alunos do curso de enfermagem do período noturno trabalham principalmente na área da saúde, durante o dia e em finais de semana. Observe-se Que há mais homens e a faixa etária é mais condizente com o fato de conseguirem estudar na Universidade à noite, concomitante ao trabalho remunerado. É visível a diferença de classificação econômica entre os dois grupos.

Nos níveis A2, B 1 e B2 estão alocados os 10 casos de depressão do Bacharelado. Os 14 casos da Licenciatura estão distribuídos nas classes A, B, C, e D com prevalência de depressão entre 28,5\% a 33,3\% nas classes B1, B2, C e D, observando-se leve tendência de diminuição da presença de depressão nas classes mais altas.

Este grupo de sujeitos (na maioria jovens e adultos jovens) encontra-se numa fase da vida de realização profissional e pessoal.
Entretanto, os índices de 15,4\% de depressão entre os estudantes do Bacharelando e 28,6\% da Licenciatura estão acima dos índices previstos para a população em geral ${ }^{(3,9,30-31)} \mathrm{o}$ Que faz sentido por ser a maioria de mulheres, com um terço em idade mais avançada entre os da Licenciatura, alguns já casados e até separados. Os dados coincidem com referências da Organização Mundial da Saúde ${ }^{(30)}$.

Os resultados deste estudo evidenciaram Que há associação da depressão com Qualidade de vida no domínio social. Os piores níveis de Qualidade de vida estão entre os alunos com depressão. Evidenciou-se também Que a média da Qualidade de vida dos alunos do Bacharelado é superior em relação aos Que freeüentam o curso noturno (Licenciatura). Entretanto, não foi o fator classe social Que marcou a diferença na Qualidade de vida e depressão entre os dois grupos de sujeitos, conforme se verificou nos testes estatísticos.

A depressão é menos freqüente entre os alunos que trabalham e não encontrou-se nenhum deles com depressão grave.

Não se encontrou diferenças estatisticamente significantes na Qualidade de vida dos sujeitos pelo fato de trabalharem ou não. A menor freqüência entre os Que trabalham, reforça a idéia de Que o trabalho pode indicar ausência de depressão. A literatura oferece suporte à este fato, ou seja, a depressão causa danos sociais (isolamento, abandono do estudo e do trabalho) $)^{(3,5,25,30-32)}$.

Quando se coloca a Questão da Qualidade de vida, a preocupação subjacente passa a ser o contexto de vida onde o sujeito está inserido pois a preocupação do pesquisador é obter elementos Que favoreçam a promoção da saúde deste segmento da população(33).

Como docentes Que têm há apenas dois anos um novo contingente noturno de acadêmicos sob sua responsabilidade este estudo mostra Que além da preocupação com os conteúdos Que devem ser ministrados, os campos de aprendizado, a didática deve star presente a preocupação com o contexto Que acompanha este grupo de alunos e seus reflexos na saúde dos mesmos. Importa saber se estes acadêmicos estão adaptando-se ao meio estudantil e como estão lidando com os fatores individuais (Que determinam as mais variadas exigências) e seus resultados na saúde do indivíduo ${ }^{(3,4,15)}$.

Os indicadores de Qualidade de vida e os indicadores sócio econômicos dão respostas gerais com resultados coletivos. Entretanto, associando-se os resultados da presença de sinais indicativos de depressão nos sujeitos do grupo selecionado para 
este estudo foram agregados elementos objetivos e subjetivos Que valorizam os resultados encontrados.

Observa-se Que nem sempre há relação direta entre trabalho e depressão, entre idade, gênero e depressão, entre fatores sociais e depressão e nem entre sensação subjetiva de saúde e depressão. Porém, os resultados dão indicações de pontos de vulnerabilidade e fragilidade Que devem ser levados em consideração pelo docente responsável pela condução pedagógica do ensino de enfermagem $^{(10,30,34)}$.

A depressão, sendo importante causa de incapacitação ${ }^{(3,5,9)}$ nem sempre é detectada, nem adequadamente tratada e orientada ${ }^{(2,6,9,35-36)}$.

Os Questionários são úteis como instrumentos de triagem, porém, os sintomas objetivos nos jovens são particularmente instáveis ${ }^{(15)}$. Assim, uma pontuação alta no teste nem sempre corresponde a um diagnóstico fechado de depressão para o jovem. Na avaliação da depressão deve-se levar em conta a sobreposição de vários fatores. Também existem evidências de Que o sucesso escolar, a capacidade do jovem para lidar para com as situações difíceis e o apoio familiar podem proteger o jovem do efeito da depressão $0^{(15)}$.

Não é possível estabelecer, a partir dos dados deste estudo, uma relação de casualidade pois muitos dos sujeitos, saindo da adolescência e entrando na idade adulta, ainda estão na fase de estabilização de seu equilíbrio, recém ingressados num dos mais cobiçados cursos de enfermagem do pais e ser o curso noturno um acontecimento recente na EERP/USP. Por outro lado, a comparação dos dois grupos de sujeitos deixou claro que há diferenças Que merecem ser consideradas.

\section{CONCLUSÕES}

Observou-se neste estudo Que há diferenças entre os alunos do curso diurno (Bacharelado) e do curso noturno (Licenciatura), levando a buscar estatísticas para encontrar algumas associações entre as diferenças.

Entre os 114 sujeitos predominam mulheres e solteiros.

No curso de Bacharelado em Enfermagem predominam os níveis econômicos A2, BI e B2, 69,6\% não trabalham, 32\% estão abaixo dos 20 anos de idade.

No curso de Licenciatura em Enfermagem, predominam os níveis B2 e C, 75,8\% têm trabalho remunerado e 32,6\% estão acima dos 25 anos.

A depressão está presente em 15,4\% dos sujeitos no Bacharelado e $28,6 \%$ na Licenciatura sendo $14 \%$ do total com depressão moderada e grave.

Os índices encontrados entre os alunos da Licenciatura são altos em relação à população em geral, inclusive com relação aos colegas do curso diurno. Ficou evidente que decresce a depressão conforme cresce o nível econômico dos alunos da Licenciatura.

Nas análises descritivas observou-se Que existe associação entre presença de depressão e Qualidade de vida, especialmente nos domínios físico, mental e social, com diferença significante no domínio social entre os 2 grupos de alunos.

$\mathrm{Na}$ análise multivariada, a auto-avaliação da saúde mental feita pelo estudante e o curso em Que está matriculado tiveram as razões de prevalência significantes. $\mathrm{O}$ aluno do curso noturno (Licenciatura) apresentou mais de duas vezes a prevalência para depressão do Que o aluno do curso diurno (Bacharelado). Além disso, aquele aluno Que reconhece Que sua saúde mental não está boa a razão de prevalência para depressão é seis vezes maior.

Conclui-se Que a percepção da saúde mental bem como a frequência a curso noturno podem ser indicadores da presença de depressão.

\section{REFERÊNCIAS}

I. Broadhead WE, Blazer DC, Georg L, Tze CK. Depression, disability days and days lost from work in a prospective epidemiologic survey. IAMA 1990; 264 (19): 2525-8.

2. Organización Panamericana de Salud. Organización Mundial de Salud. Programa de Salud Mental. División de Promoción de la Salud. Modelo para la capacitación de la enfermera general en la identificación y manejo de los transtornos afectivos. Generalista I. Genebra: OMS; 1999.

3. Maj M, Sartoius N. Transtornos depressivos. Porto Alegre: Artmed; 2005.

4. Rosenbaum IF, Hylan TR. O custo dos transtornos depressivos: uma revisão. In Maj M, Sartorius N. Transtornos depressivos. Porto Alegre: Artmed; 2005.

5. Dalgalarrondo P. Psicopatologia e semiologia dos transtornos mentais. Porto Alegre: Artes Médicas; 2007.

6. Katzelnick DI, Koback KA, Greist JH, Jefferson JW, Henk IJ. Effect of primary care treatment of depression on service use by hight medical expenditures. Psych Serv 1997; 48(1): 59-64.

7. Okasha A. Subdiagnóstico da depressão seu impacto nas comunidades. In Maj M, Sartorius N. Transtornos depresivos. Porto Alegre: Artmed; 2005.

8. Stefanis CN, Stefanis NC. Diagnósticos dos transtornos depressivos: uma revisão. In Maj M, Sartorius N. Transtornos depressivos. Porto Alegre: Artmed; 2005. p. 13-76.

9. Levav I, editor. Temas de salud mentall en la comunidad. Washington: OPAS/OMS; 1992

10. Adams SM, Partee DI. Integrating psychosocial rehabilitation in a community-based faculty nursing practice. I Psych Nurs 1998; 36(4): 24-8.

11. Pitta A, organizadora. Reabilitação psicossocial no Brasil. São Paulo: Hucitec; 2001.

12. Tousend MC. Enfermagem psieuiátrica: conceitos de cuidados. Rio de Janeiro: Guanabara Koogan; 2002.

13. Kopelowicz MD, Liberman RP. Integrating treatment with rehabilitation for persons with major mental illnesses. Psych Serv 2003; 54 (1 1): 1491-8.

14. Harrington R, Wittaker I, Shoebridge P, Campbell F. Systematic review of efficacy of cognitive behaviour therapies in child and adolescent depressive disorder. Brit Med I 1998; 316 (7144): 1559-63.

15. Harrington R. Transtornos depressivos em crianças e adolescentes: uma revisão. In Maj M, Sartorius N. Transtornos depressivos. Porto Alegre: Artmed; 2005. p. 191-250.

16. Franco GP, Barros ALPL, Nogueira-Martins LA. Qualidade de vida e sintomas depressivos em residentes de enfermagem. Rev Latino-am Enfermagem 2005; 13(2): 139-44.

17. Furegato ARF, Silva EC. A doença mental vivida por um paciente psiQuiátrico: suas percepções. Esc Anna Nery Rev Enf 2006; 
10: 652-59.

18. Saupe R, Nietche EA, Cestan ME, Giorgi MDM, Krahi M. Qualidade de vida dos acadêmicos de enfermagem. Rev Latinoam Enfermagem 2004; 12(4): 636-42.

19. Santos TM, Almeida AO, Martins HO, Moreno V. Aplicação de instrumento de avaliação do grau de depressão em universitários no interior paulista, durante a graduação de enfermagem. Acta Sci Health Sci 2003; 25(2): 17 I-6.

20. Pasquali L. Instrumentos psicológicos: manual prático de elaboração. Brasília: Prática Gráfica; 1999.

21. Associação Brasileira de Empresas de Pesquisa. Critério de Classificação Econômica Brasil. São Paulo: ABEP; 2005.

22. Beck AT, Ward CH, Mendelson M, Mock J, Erbaugh J. An inventory for measuring depression. Arch GenPsych 1961; 4: 551-571.

23. Gorenstein C, Andrade L. Inventário de depressão de Beck: propriedades psicométricas da versão em português. Rev Psie Clin 1998; 25(5): 245-50.

24. Fleck MPA, Lousada S, Xavier M, Chachamovich E, Vieira G, Santos L, Pinzon V. Aplicação da revisão em português do estudo de avaliação de Qualidade de vida da Organização Mundial da Saúde (WHOQOL 100). Rev Saúde Pública 1999; 33(2): 198-205.

25. Fleck MPA. Diretrizes da Associação Médica Brasileira para o tratamento da depressão (versão integral). Rev PsiQ Clin 2003; 25(2): 114-22.

26. Siegel S, Castellan Jr NJ. Estatística não paramétrica para ciências do comportamento. Porto Alegre; Artmed; 2006.

27. Barros AJD, Hirakata VN. Alternatives for logistic regression in cross-sectional studies: an empirical comparison of models that directly estimate the prevalence ratio. BMC Med Res Meth 2003; $3: 21$

28. Hamilton LC. Statistics with STATA. Toronto: Doxbury-Thomson Learning; 2003.

29. Wetterich NC, Melo MRAC. Sociodemographic profile of undergraduate nursing students. Rev Latino-am Enfermagem 2007; 15(3): 404-10.

30. World Health Organization (WHO). Gender and women's health. Geneve: WHO; 2008. [cited 2008 april 4]. Available from: http://www.who.int/mental_health/prevention/genderwomen/en/ index.html

31. World Health Organization (WHO). Suicide prevention. Geneve: WHO; 2008. [cited 2008 april 4]. Available from: http:// www.who.int/mental_health/prevention/suicideprevent/en/ index.html

32. Willians RA, Strasser PB. Depression in the workplace: impact on employees. AAOHN J 1999; 47(11):526-539.

33. Minayo MCS, Hartz ZMA, Buss PM. Qualidade de vida e saúde: um debate necessário. Cien Saúde Coletiva 2000; 5(1):19-31.

34. World Health Organization. Atlas: nurses in mental health. Geneve: WHO; 2007.

35. McQuaid IR, Stein MB, Laffaye C, McCahill ME. Depression in a primary care clinic: the prevalence and impact of a unrecognized disorder. J Affe Dis 1999; 55(1): 1-10.

36. Silva MCF, Furegato ARF, Costa Ir ML. Depressão: pontos de vista e conhecimento de enfermeiros da rede básica de saúde. Ribeirão Preto(SP): Rev Latino-am Enfermagem 2003; I I (I): 7-13. 